\title{
Mass spectrometry and proteomics: hitting the mark
}

Nathan Blow

\author{
Mass spectrometry instrumentation has made strides in recent years in terms of dynamic range and \\ sensitivity, putting researchers in a better position to use the technology to tackle the challenges of \\ disease biomarker discovery and validation.
}

At the American Society for Mass Spectrometry (ASMS) meeting in June 2008 , mass spectrometry instrumentation aimed at identification of protein biomarkers - proteins indicative of a disease or disease state-appeared to be a recurring theme among instrument manufacturers.

Waters Corporation in Milford, Massachusetts, USA, introduced a new tandem quadrupole mass spectrometry system, called the Xevo TQ MS, along with an associated software package, both designed to move from the biomarker discovery stage towards the verifiction and validation of target proteins. Agilent Technologies in Foster City, California, USA, introduced their new 6400 series of triple quadrupole liquid chromatography-mass spectrometry systems with increased sensitivity. "The advantage of doing a validation assay on a tandem quadrupole is the improved dynamic range and sensitivity," notes James Langridge, director of proteomics business development at Waters Corporation.

"I think we are at a promising time in the utilization of mass spectrometry toward finding new disease biomarkers," says Jan Schnitzer from the Sidney Kimmel Cancer Center in San Diego. But the instrument performances in terms of dynamic range and sensitivity remain limiting for the challenging task of discovering biomarkers in complex samples such as serum or plasma. "The complexity of the blood is not just a few hundred proteins: it is a lot more diverse than that. And when you get into that level of molecular diversity, it was, and still is to some extent, beyond our means," says Schnitzer. To circumvent these limitations, researchers are increasingly turning to

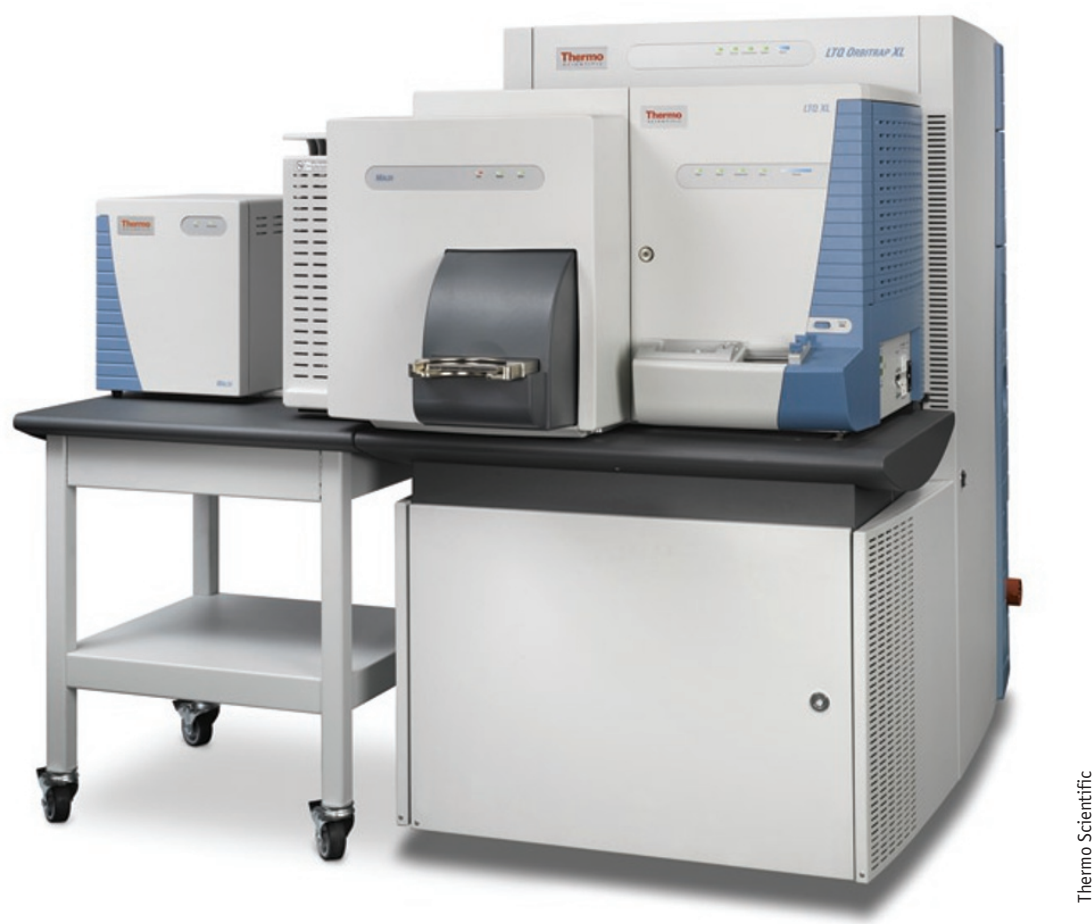

Recently, Thermo Scientific added MADLI and electron transfer dissociation (ETD) capabilities to their Orbitrap systems.

novel methods and targeted approaches to use the instrumentation more effectively.

\section{Decreasing the range}

"I think we are at a dynamic range of about $10^{4}$," says Matthias Mann from the Max Planck Institute of Biochemistry in Martinsried, Germany, speaking about the current crop of mass spectrometers. Human plasma or serum, however, contain proteins in a range of concentrations that, by some estimates, covers 12 orders of magnitude.

From the instrument side, there seems to be no easy solution to increasing the dynamic range. "I don't see anything that will get us to $10^{8}$ or $10^{10}$, so that is out of the range of the current technology," says Mann. Waters Corporation's Langridge thinks the dynamic range of a mass spectrometer is probably limited by the ionization techniques used, noting that electrospray ionization provides the capacity to measure only about 5 orders of magnitude.

Even though it appears that the instrumentation alone will not evolve sufficiently in the near future to cover the entire range of concentrations, researchers are devising methods to get around the problem. For a 
start, Mann notes that some simple samplepreparation approaches, such as selectively capturing certain classes of proteins for analysis or removing the most abundant proteins, help improve the dynamic range of current instruments. "Your only choice if you have a limited machine is to break up all the parts and then perform the analysis," says Schnitzer.

\section{Discovering the least complicated}

An effective, if simple, approach is to tackle a less challenging sample in the search for disease biomarkers. "Plasma is very difficult from a proteomic standpoint," says Mann, "but for tissue I think the prospect is quite good that you can do this with the current technology." Tissue samples tend to contain proteins in ranges of concentrations that are not as extreme as plasma and present other potential advantages. "People believe that tissue is closer to the site of the disease, and that therefore the level of the marker might be potentially elevated in the tissue," says Waters' Langridge.

Instrument builders, responding to this potential new avenue, have developed new systems directed toward mass spectrometry-based tissue analysis. Bruker Daltonics, located in Bremen, Germany, has a system based on matrix-assisted laser desorption/ ionization (MALDI) tandem time-of-flight (TOF/TOF) technology, called ultraflex III TOF/TOF, which is designed for tissue imaging, including the protein mass range.
Earlier this year, Thermo Scientific, located in Waltham, Massachusetts, USA, introduced a MALDI ionization source on its hybrid LTQ Orbitrap mass spectrometer, which can also be used to perform tissue imaging studies.

Detlev Suckau, head of MALDIapplications development and proteomics at Bruker Daltonics, has worked with other scientists to identify biomarkers using mass spectrometry-based tissue imaging. Suckau's approach has been to first obtain a set of tissue images using the ultraflex III TOF/TOF system, then perform hierarchical clustering of the tissue samples, followed by pathologists' assignments of the health state within each cluster and finally identify the markers that group with these assignments.

"It was surprising how short the time was to develop intelligent estimates of candidates," he notes-much faster than to reach the equivalent level of certainty with serum samples. Still, he thinks it is probably too early to judge the true potential of a technology like mass spectrometry-based tissue imaging for biomarker discovery as it has only been in researcher's hands for a year or two.

\section{Straight to the target}

Some researchers, like Ruedi Aebersold, have not given up on the challenge of looking for cancer biomarkers in serum samples, but this requires work-arounds. Attempting

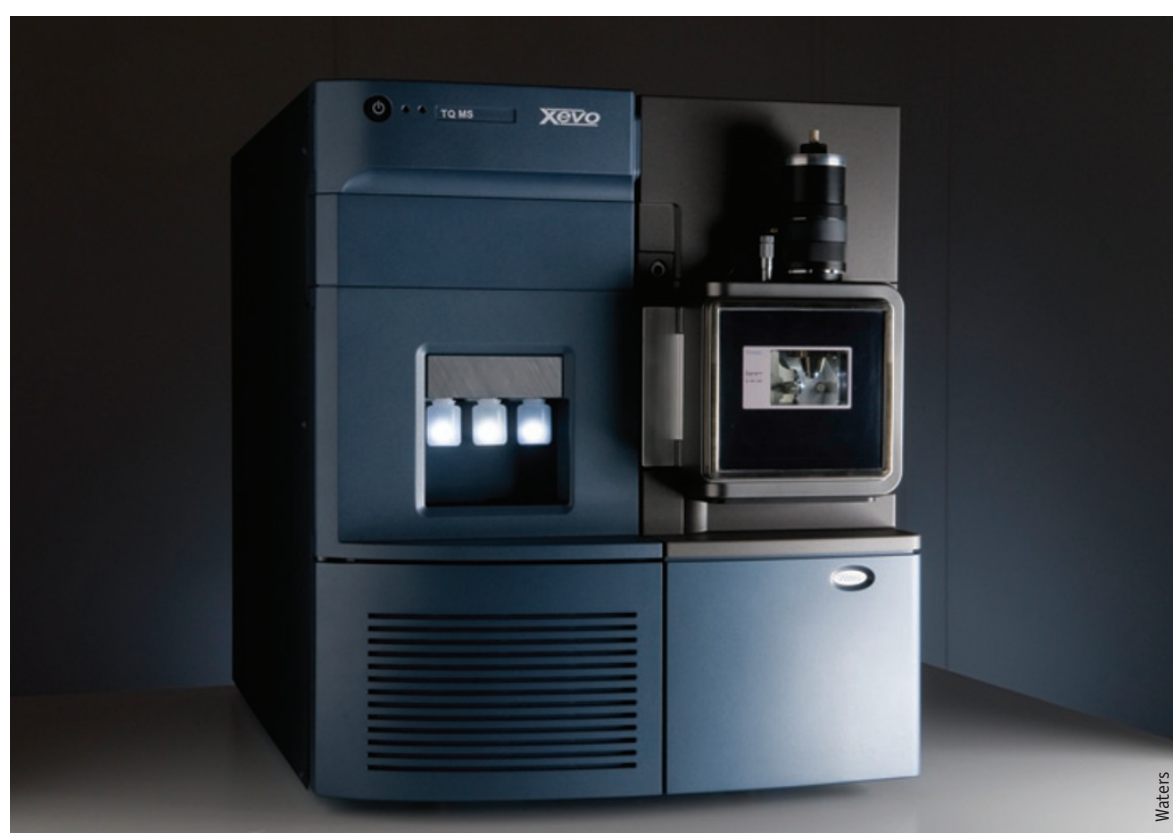

The new Xevo TQ MS from Waters is designed to aid in the process of biomarker validation. 
to compare whole serum samples from diseased and healthy individuals, Aebersold found out that the mass spectrometers were not measuring down into the necessary nanogram per milliliter concentration range, or if they did, it was with an enormous effort, requiring multiple mass spectrometry runs-many hundreds of hours of machine time - to completely compare just on two samples. "I came to the conclusion that this is not the way to go," says Aebersold.

Instead, his group is exploring a 'targeted proteomics' approach to protein biomarker discovery. "We will go and look in a targeted manner for specific proteins, or sets of proteins, that we think might be related to sets of disease," says Aebersold. They initially rely on comparisons between affected and normal individuals using independent approaches, such as microarrays, literature searches and systems-level analysis to identify candidate proteins. They then perform

을 multiple-reaction monitoring (MRM) measurements, in triple quadrupole mass spectrometers, to detect and quantify the

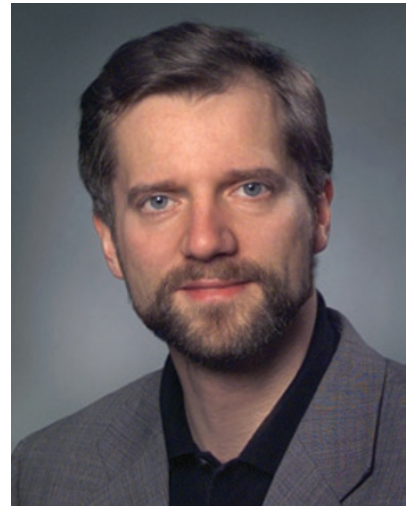

Matthias Mann thinks label-free approaches to quantitative mass spectrometry could help in biomarker discovery.

sets of proteins in serum samples-looking for differences between disease and control cases. By using MRM, the researchers 'instruct' the instrument to specifically monitor selected ion masses and ignore other ions. This permits very selective and sensitive detection and analysis.
Of course, using such a targeted approach, you can only find the biomarkers you are specifically looking for. But there are several benefits to reducing the number of proteins examined by mass spectrometry. "You eliminate a huge amount of noise-person-toperson and other noise-because most of the proteins you are not going to look at," Aebersold says. He adds that targeted mass spectrometry methods are orders of magnitude more sensitive than untargeted discovery-comparison methods, especially if faced with very complicated samples like serum.

Following a similar path, the US National Cancer Institutes' Clinical Proteomic Technology Assessment for Cancer (CPTAC) program is also investing in a targeted, candidate-based approach. "A candidate-based method is much more robust and quantitatively precise," says Steven Carr, director of the proteomics platform at the Broad Institute in Cambridge, Massachusetts, USA, and a CPTAC investigator. CPTAC worked with the cancer research community to identify approximately 1,000 candidate 


\section{BOX 1 STANDARDS AND PRACTICES}

In January 2006, the US National Cancer Institute started the pilot phase of the Clinical Proteomics Technology Assessment for Cancer (CPTAC), a five-year, \$104 million program to explore the application of proteomics to clinical cancer diagnostics.

Steven Carr, a principal investigator for one of the CPTAC working groups at the Broad Institute, says that proteomics has yet to substantially contribute to the development of clinical diagnostics. "The technologies are not the fault; it is the lack of clear understanding about what the technology base is capable of, how best to prepare clinical samples for these platforms and what samples are best for proteomic studies," says Carr. This is leading Carr, at the Broad Institute, and CPTAC groups at Memorial SloanKettering Cancer Center in New York; Purdue University in West Lafayette, Indiana; University of California, Francisco-Lawrence Berkeley National Laboratory-Buck Institute in California; and Vanderbilt University Medical School in Nashville, Tennessee to create a standardized pipeline for protein biomarker discovery and verification.

The five CPTAC groups are collaborating to test samples across labs and establish standardized procedures in two areas: unbiased discovery proteomics and candidate verification by MRM. By keeping all that they possibly can consistent-mass spectrometry systems, workflows and standard operating procedures - the five groups are systematically investigating where sources of variation arise. "In the case of unbiased discovery, the teams will be using up to 100 proteins spiked into complex biological backgrounds such as cell lysates to ask how reliably and reproducibly are proteins present at different abundances detected, how robustly differences in levels of specific proteins between samples can be identified [simulating actual biomarker discovery], and what the false discovery rates are. In the case of targeted verification using MRM, we are using stable isotope-labeled peptides to quantify proteins spiked into plasma and establish reproducibility, linearity of response and limits of detection across seven instruments in five different research groups," explains Carr.

CPTAC is now moving toward the next phase of biomarker identification: a pipeline for verification at large scale. Leigh Anderson from the Plasma Proteome Institute in Washington, DC says the pipeline has to be robust and well validated. "If a marker is there, we need a pipeline that we are confident will find it," he says, as he predicts an attrition rate of $95-98 \%$ of candidates during the clinical validation phase.

Both Anderson and Carr think the biggest challenge during the verification phase will be dealing with the limited sensitivity of current mass spectrometry instrumentation. They are banking on instrument manufacturers to deliver systems with a 5-10-fold increase in sensitivity to help solve the issue. protein biomarkers related to cancer based on prior independent observations. A subset of these candidates will be put to the test using a high-throughput verification pipeline being developed by CPTAC participants (see Box 1).

The newly introduced triple quadrupole systems and software might help expand the usage of targeted approaches. Waters' Xevo TQ MS features the ability to perform tandem mass spectrometry (MS/MS), while at the same time acquiring quantitative MRM data, which was not possible with previous tandem quadrupole instruments. This allows the users to select proteins of interest by MS/MS and follow up

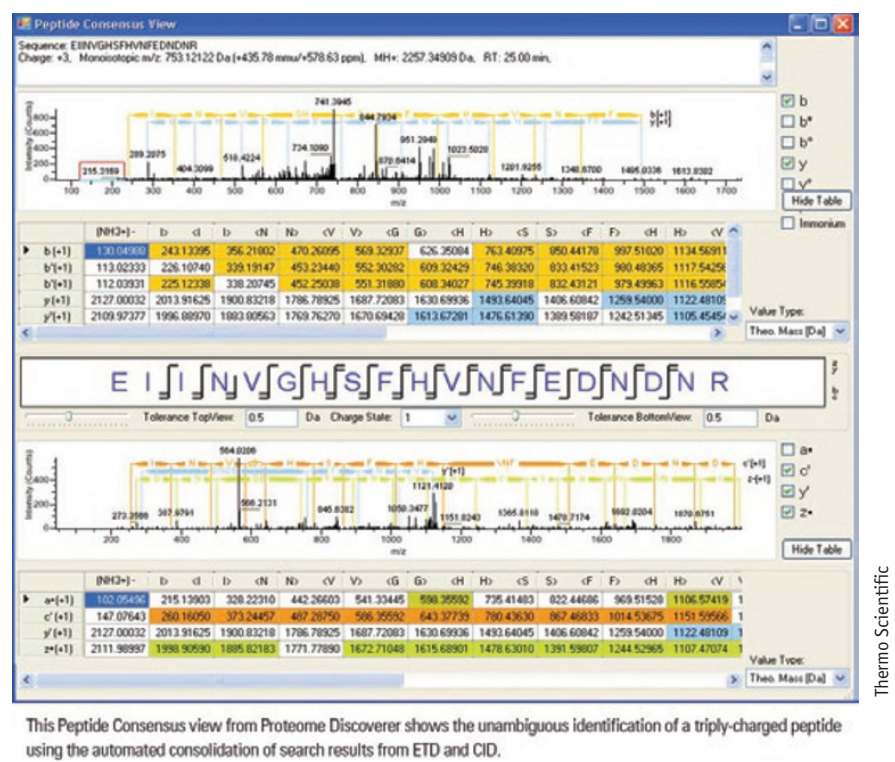

New software programs and tools, such as the recent introduced Proteome Discoverer from Thermo Scientific, are making the interpretation of mass spectrometry data easier for users. with MRM analysis of the selected ions in the same run. The system takes advantage of the new 'VERIFYE' software, associated with the Xevo TQ MS, which examines all the peptides and proteins identified from the MS/MS analysis phase, and finds the most ionizing peptides and the best fragments to monitor during a targeted assay.

\section{Numbers game}

"I think quantitative mass spectrometry is a very exciting field and a lot has been achieved in the past couple years on both the label and label-free side," says Bruker Daltonics' Suckau. The rise of quantitative mass spectrometry within the proteomics community is the result of developers working hard in recent years to get both label and label-free methods and kits into the hands of researchers.

On the 'label side', Thermo Scientific recently introduced a new tandem mass tag kit, called TMT, for use in labeling experiments. The kits were developed by Proteome Science located in Cobham, UK. They contain isobaric tandem mass tags with an amine reactive group, a mass normalizing linker, a cleavable bond and a series of reporter ions. With these reagents, researchers can specifically label proteins and peptides in a sample to obtain relative or absolute quantification. 
On the 'label-free side', Mann points directly to advances in instrumentation as the catalyst for the increased popularity of quantitative approaches. "We have the Orbitrap, which is good for label-free approaches, and the advanced TOF instruments also have good resolution; that has been one good step," says Mann. "In general I think label-free looks very interest-

ing, especially in the context of biomarker discovery."

Lester Taylor, director of marketing for the mass spectrometry and life sciences group at Thermo Fisher, concurs on the importance of resolution. "There are a lot of instruments that promote mass accuracy, but it is not all about mass accuracy. One of the keys is to have resolution that allows you to be sure that the peak you are monitoring is isolated and well resolved from any interference," says Taylor.

Aebersold's group uses both label and 을 label-free methods for their targeted pro- teomics approaches. His group prefers to use isotope-coded affinity tag labeling or iTRAQ labeling for validation of a potential biomarker. "For the detection of the targeted peptides in serum, we use stable isotope labeling reference peptides to determine the absolute quantity of serum proteins," he says, "for the simple reason that you want to accurately quantify the peptides in the sample and read out the absolute concentrations." But to initially establish their list of proteins of interest during the discovery phase, his group uses label-free methods, which are less expensive and can be performed at higher throughput.

\section{Validation and beyond}

"You do have to look across a large number of samples to be able to see if a biomarker will have clinical utility," says Langridge, addressing another issue in protein biomarker validation: sheer sample numbers. Because of the diversity of the human population, statistically significant validation of a candidate protein biomarker could require examining hundreds of patient samples. At this stage of development, however, the higher-resolution instruments necessary for validation tend not to be amenable to high throughput.

Carr thinks that such throughput issues can be solved in the coming years with the development of robust high-throughput pipelines. He thinks most of the bottleneck during this validation phase will not come from the mass spectrometer itself but from the sample preparation. The CPTAC members, along with an increasing number of commercial developers, are working on technologies to increase throughput for all portions of the discovery and validation process.

Nathan Blow is the technology editor for Nature and Nature Methods (n.blow@boston.nature.com). 


\section{SUPPLIERS GUIDE: COMPANIES OFFERING MASS SPECTROMETRY INSTRUMENTATION AND ACCESSORIES}

\section{Company}

Adron Systems LLC

Agilent

Alcott Chromotography

Analytica of Branford, Inc.

Applied Biosystems

Astra Analytical

Bergmann Messgerate Entwicklung KG

BioTrove

Bruker Daltonics

Comstock Incorporated

De Tech

Evans Analytical Group

Expression Pathology

Ferran Scientific

Full Spectrum Analytics

$G B$ Scientific

GE Healthcare

Hitachi Instruments

HT Laboratories

Hybrigenics

In Process Instruments

Invitrogen

Jeol

Jordan TOF Products, Inc.

Kore Technology

Kratos Analytical

LEAP Technologies

Leco Corp.

M-Scan

(2) Metabolic Solutions Inc. Monitor Instruments

Mspec Group

Omega Engineering, Inc.

Omni International

Perkin Elmer

Photonis

Prolab Resources

Restek

Scientific Instrument Services, Inc.

Shimadzu Scientific Instruments

Sigma Aldrich

SGE Analytical Sciences

Thermo Electron Corp.

Varian

Waters

\section{Web address}

http://www.adronsystems.com/

http://www.agilent.com/

http://www.alcottchromatography.com/

http://www.aob.com/

http://www.appliedbiosystems.com/

http://www.astraanalytical.com/

http://www.bme-bergmann.de/

http://www.biotrove.com/

http://www.bdal.com/

http://www.comstockinc.com/

http://www.detechinc.com/

http://www.eaglabs.com/

http://www.expressionpathology.com/

http://www.ferran.com/

http://www.fullspectrum-inc.com/

http://www.gbscientific.com/

http://www.gehealthcare.com/

http://www.hitachi.com/

http://www.ht-labs.com/

http://www.hybrigenics.com/

http://www.in-process.com/

http://www.invitrogen.com/

http://www.jeol.com/

http://www.rmjordan.com/

http://www.kore.co.uk/

http://www.kratos.com/

http://www.leaptec.com/

http://www.leco.com/

http://www.m-scan.com/

http://www.metsol.com/

http://www.monitorinstruments.com/

http://www.mspecgroup.com/

http://www.omega.com/

http://www.omni-inc.com/

http://www.perkinelmer.com/

http://www.photonis.com/

http://www.prolabresources.com/

http://www.restek.com/

http://www.sisweb.com/

http://www.shimadzu.com/

http://www.sigmaaldrich.com/

http://www.sge.com/

http://www.thermo.com/

http://www.varianinc.com/

http://www.waters.com/ 


\section{Erratum: Mass spectrometry and proteomics: hitting the mark}

\section{Nathan Blow}

Nat. Methods 5, 741-747 (2008); published online 1 August 08; corrected after print 29 September 2008

In the version of this article initially published, Box 1 incorrectly listed the University of California, Berkeley as one of the five Clinical Proteomic Technology Assessment for Cancer (CPTAC) groups. The University of California, San Francisco-Lawrence Berkeley National Laboratory-Buck Institute is the correct designation for this CPTAC group. This error has been corrected in the HTML and PDF versions of the article. 